\title{
Persistent elevation of aminotransferases in liver transplant in association with chronic norovirus infection
}

\author{
Ammar Abdulaziz Khayat ${ }^{1,2}$ and Grzegorz W Telega' \\ 'Department of Pediatric Gastroenterology, Medical College of Wisconsin, Milwaukee, WI, USA; ${ }^{2}$ Department of Pediatrics, College of \\ Medicine, Umm Al Qura University, Al Abdeyah, Makkah, Saudi Arabia
}

Dear Editor,

Many human pathogenic viruses cause hepatocyte injury and inflammation resulting in increase in serum aminotransferase levels as a manifestation (clinical hepatitis). The mechanism of liver injury for some of those viruses is not fully understood. Hepatotropic viruses with the prototype being hepatitis B cause liver injury not by direct cytopathic effects, but rather by forcing hepatocytes to express viral proteins that in turn trigger the host's own immune system, in the form of $T$ cell activation (predominantly CD4+, CD25+ T reg) resulting in hepatocyte apoptosis through expression of pro-apoptotic molecules and initiation of extrinsic apoptosis cascade. ${ }^{1}$

Non-hepatotropic viruses (e.g., influenza) on the other hand, cause liver injury by inducing overexpansion of viral antigen-specific T cells (CD8+), with subsequent entrapment by Kupffer cells, resulting in foci of inflammation and apoptosis, with no direct inoculation of hepatocyte. ${ }^{2}$

Elevated serum aminotransferase levels have been linked to norovirus related acute gastroenteritis in several reports. ${ }^{3-6}$ Whether Norovirus directly inoculates hepatocytes or causes a "collateral damage" phenomenon (as seen with influenza) is unknown. Nevertheless, dissemination of the virus past intestinal tissue into mesenteric lymph nodes, serum, and spleen have been documented in animal studies. ${ }^{7}$ Acute gastroenteritis related "extra-intestinal" manifestations including benign infantile convulsions, encephalopathy, disseminated intravascular coagulation have been linked to presence of Norovirus past intestinal tissue. ${ }^{7}$ Typically, aminotransferase levels normalize within 4 weeks of onset of acute gastroenteritis in otherwise healthy subjects. ${ }^{3}$ Whether this applies to immunocompromised hosts is uncertain, especially since they may shed the virus or have clinical disease for years. ${ }^{8}$

We present two cases of liver transplants, who present with chronic norovirus infection/shedding with chronic hepatitis and persistently elevated serum aminotransferases (beyond 4 weeks) not attributable to any other etiology.

\section{CASE 1}

A three-year-old girl was diagnosed with hepatoblastoma at the age of 11 months. She received chemotherapy, then orthotopic liver transplant with duct to duct anastomosis and biliary stent placement 2 months later. Donor was cytomegalovirus (CMV) and Epstein-Barr virus (EBV) negative. Post-operative course was uncomplicated, and her aminotransferase levels normalized within

\section{Abbreviations:}

ALT, alanine aminotransferase; AST, aspartate aminotransferase; CMV cytomegalovirus; C. diff, Clostridium difficile; EBV, Epstein-Barr virus; HHV, human herpes virus; IVIG, intravenous immunoglobulin

\section{Corresponding author: Ammar Abdulaziz Khayat}

Department of Pediatric Gastroenterology, Medical College of Wisconsin, 8701 W Watertown Plank Road, Milwaukee, WI 53226, USA

Tel: +1-414-955-8296, Fax: +1-414-266-3676

E-mail: Ammarkhayat307@hotmail.com

https://orcid.org/0000-0002-4819-9552

Received : Feb. 4, 2019/ Accepted : Feb. 21, 2019

Copyright $\odot 2019$ by Korean Association for the Study of the Liver

This is an Open Access article distributed under the terms of the Creative Commons Attribution Non-Commercial License (http://creativecommons.org/licenses/by-nc/3.0/) which permits unrestricted non-commercial use, distribution, and reproduction in any medium, provided the original work is properly cited. 
two weeks of transplant. Standard of care and immunosuppression (including tacrolimus) protocols were followed. Five months later she was found to have elevated aminotransferase levels (aspartate aminotransferase [AST] 84, alanine aminotransferase [ALT] 119 IU/L). She was anemic with hemoglobin of eight with low white blood cell count that was attributed to chemotherapy, and otherwise her labs were essentially normal. At that point she was having diarrhea coinciding with elevated transaminases (up to 10 times a day, watery non-bloody) and non-bilious nonbloody vomiting. Stool testing was positive for Norovirus (Verigene ${ }^{\circledast}$, Luminex Corporation, Austin, TX, USA; Enteric Pathogens Nucleic Acid Test). The rest of the panel was negative. Clostridium difficile (C. diff), blood and stool adenovirus and enterovirus, human herpes virus (HHV)-6, CMV, EBV, nasopharyngeal swabs were all negative as well. She underwent a liver biopsy at the time that showed few portal tracts with lymphocytic infiltration invading adjacent lobules, with slight sinusoidal and lobular eosinophils and hepatocyte ballooning/dropout, acute rejection was ruled out (Fig. 1A). Liver tissue viral culture was negative. Liver ultrasound with Doppler showed stable waveforms. Testing for donor-specific antibodies (for antibody mediated rejection), and liver ultrasound/ doppler were negative. Elevated aminotransferase levels were thus attributed to norovirus infection. Subsequently, stool testing for norovirus was persistently positive in four different occasions with the most recent being 10 months from first infection (15 months post-transplant). She did have mild baseline symptoms of loose stools up to 4 times daily and daily vomiting.

Her aminotransferases became persistently elevated over the course of 10 months (ALT range 49-75 with peaks up to $400 \mathrm{IU} / \mathrm{L}$, AST normal to 60 with peaks to $700 \mathrm{IU} / \mathrm{L})$. She underwent multiple liver biopsies that showed similar findings to the first one.

Subsequently, intravenous immunoglobulin (IVIG) at a dose of $1 \mathrm{~g} / \mathrm{kg}$ was administered. A total of 3 doses 1 week apart were given. Her aminotransferase levels showed improvement after 3rd dose (ALT dropped from 400 to $200 \mathrm{IU} / \mathrm{L}$ ), and later complete normalization in 5 months (21 months following transplant).

\section{CASE 2}

An 8-year-old boy underwent liver transplantation for the diagnosis of congenital hepatic fibrosis and Caroli's disease. Post-operative course was uncomplicated. Aminotransferases rose 3 months later with ALT in 90 and AST in $60 \mathrm{IU} / \mathrm{L}$. He did have symptoms of diarrhea and vomiting at the time.

Liver biopsy did show lobular inflammation with patchy portal inflammation with lymphocytes, eosinophils and histiocytes, but no plasma cells, also hepatocyte dropout (Fig. 1B). Rejection was ruled out. Viral tissue cultures and stainings were also negative. Similar to case 1, stool testing was positive for Norovirus (Verigene ${ }^{\circledast}$, Luminex Corporation; Enteric Pathogens Nucleic Acid Test). The rest of the panel was negative. $C$. diff, blood and stool adenovirus and enterovirus, HHV-6, CMV, EBV, nasopharyngeal swabs were all negative as well. His stools studies persistently tested positive for norovirus in four different occasions, up to a year following transplant. Aminotransferase levels remained ele-
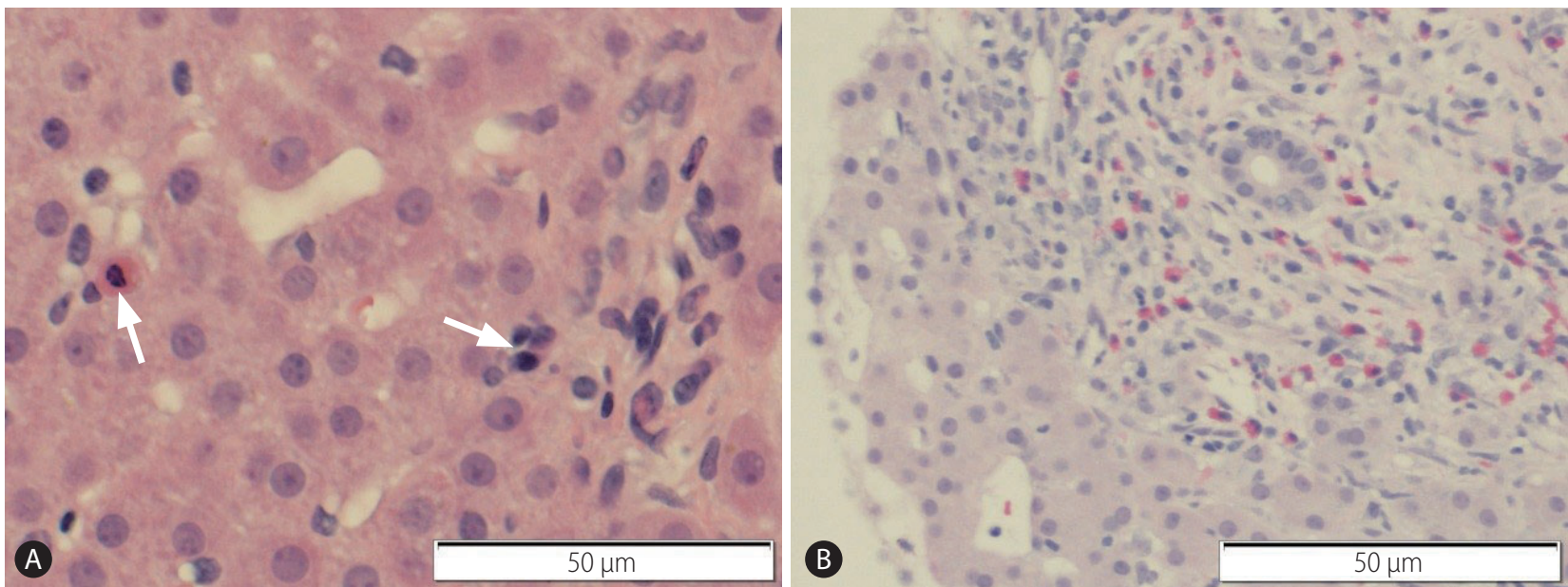

Figure 1. Liver biopsies obtained during the course of persistent elevation of aminotransferases and norovirus infection. (A) Hematoxylin and Eosin stain showing hepatocyte dropout (apoptosis) (left arrow), with lymphocytic infiltrate (right arrow). (B) Hematoxylin and Eosin stain Lobular inflammation with mixed lymphocytic and eosinophilic infiltrate. 
vated during the course (peak ALT $400 \mathrm{IU} / \mathrm{L}$, peak AST $300 \mathrm{IU} / \mathrm{L}$ ). Multiple liver biopsies showed findings similar to the first biopsy (lobular inflammation with patchy portal inflammation with lymphocytes, eosinophils and histiocytes, but no plasma cells, also hepatocyte dropout), with one of them showing an additional finding of neutrophilic microabscess consistent with a viral hepatitis pattern.

His stool studies persistently tested positive for norovirus in four different occasions, up to a year following transplant. However, his diarrhea lasted less than 2 weeks following initial infection, with subsequent intermittent episodes lasting less than 3 days. Therefore, he was considered a chronic "carrier".

Eventually, aminotransferase levels improved after lowering immunosuppression (in the form of decreasing tacrolimus trough levels), and he was able to clear the virus.

\section{DISCUSSION}

Chronic diarrhea caused by Norovirus infection and persistent shedding in immunocompromised and transplant patients have been observed in many studies. ${ }^{8,9}$ In fact, It is one of the most common infectious agents isolated from patients with chronic diarrhea in this setting, followed by C. diff and CMV. In an adult study of solid organ transplant recipients; norovirus was isolated in $35 \%$ of subjects presenting with chronic diarrhea, compared to C. diff and CMV that were isolated in $25 \%$, in $12 \%$ of cases respectively. Diarrhea due to Norovirus was associated with the most protracted course; up to 241 days compared to 71 days in the control group. ${ }^{9}$

Many non-hepatotropic viruses can potentially induce liver injury, possibly by an indirect pathway. The exact mechanism of injury is not well understood, but influenza induced hepatitis in animal studies showed that when aminotransferase levels were elevated, viral genome was absent in hepatocytes, while strongly present in lung tissue; indicating a "collateral damage" phenomenon. ${ }^{2}$ Interestingly, liver histology did demonstrate lymphocytic infiltration (CD8+ T cell predominant) in the influenza-infected mice along with apoptotic bodies. ${ }^{2}$ This is in line with the lobular inflammation and hepatocyte dropout seen in our cases, a picture that is commonly found in viral induced hepatitis. ${ }^{10}$

Norovirus can potentially be one of those viruses that cause "collateral damage" phenomenon rather than direct cytopathic or specific (CD4+ T reg cell activation) immune dependent necroinflammation seen with hepatotropic viruses. Regardless of the mechanism, many studies consistently demonstrated a relationship between Norovirus related acute gastroenteritis and elevation in serum aminotransferases. ${ }^{3-6}$ Aminotransferase levels completely normalized with the resolution of diarrhea or within maximum of 4 weeks of symptom onset. Unfortunately, isolating Norovirus from liver tissue is challenging as there's no commercially available test for Norovirus tissue culture or tissue polymerase chain reaction, making it difficult to understand the exact mechanism of liver injury.

The prolonged duration of viral effect on hepatocytes (or enterocytes in case of prolonged diarrhea) in transplant status may be explained by alteration in T cell function with immunosuppressive drugs, specifically calcinurin inhibitors. Tacrolimus has been documented to inhibit T cell cytotoxicity, and alter the ratio of Th1 (CD45RC high)/Th2 (CD45R low) preferentially inhibiting Th1 cells. Some animal studies showed that "CD45R low" T cells were linked to uveitis, and immune regulation in Graft Versus Host Disease. ${ }^{11,12}$ Whether alteration in Th1/Th2 ratio is directly related to the prolonged effect of norovirus on hepatocytes is not clear.

Treatment of chronic norovirus infection in immunocompromised host is not well established. Decreasing immunosuppression dosage, Nitazoxanide, IVIG, or oral immunoglobulin may be considered; although evidence of their effectiveness is limited, and further studies are necessary to determine optimal treatment. $^{13,14}$

Our cases had documented persistent norovirus positivity in stools, along with high aminotransferase levels lasting beyond 4 weeks, and biopsy findings compatible with chronic viral hepatitis, while essentially having negative comprehensive viral workup. This is an interesting finding since previously reported association between Norovirus and elevated aminotransferases was limited to the 4 week mark or the acute phase of gastroenteritis. To our knowledge, this has not been previously reported. Those cases present a unique and interesting observation that warrants further studies and improved virus isolation techniques from liver tissue to determine the exact mechanism of injury.

\section{CONCLUSION}

Chronic "transaminitis" and hepatitis can be observed in association with chronic Norovirus infection and carrier state in the setting of liver transplant, albeit the exact mechanism is unknown. It is reasonable to consider chronic norovirus infection or carrier state in the differential when evaluating liver transplant 
patients presenting with elevated aminotransferases when other common etiologies have been excluded, especially since the empiric use of steroids for presumed rejection can further prolong the infection.

\section{Authors' contribution}

Ammar Abdulaziz Khayat wrote the manuscript. Grzegorz W Telega helped with editing it. All authors approved the final version of manuscript.

\section{Acknowledgement}

James Southern, MD, provided pathology figures and legends.

\section{Ethical statement}

The study was approved by the Children's Hospital of Wisconsin's institutional review board (IRB) with waivers for HIPAA and consent. Approval ID is Children's Hospital of Wisconsin IRB (FWA00001809) Board \#1 (Registration IRB00002082) and Board \#2 (Registration \# IRB00006080).

\section{Conflicts of Interest}

The authors have no conflicts to disclose.

\section{REFERENCES}

1. Nakamoto Y, Kaneko S. Mechanisms of viral hepatitis induced liver injury. Curr Mol Med 2003;3:537-544

2. Polakos NK, Cornejo JC, Murray DA, Wright KO, Treanor JJ, Crispe IN, et al. Kupffer cell-dependent hepatitis occurs during influenza infection. Am J Pathol 2006;168:1169-1178; quiz 1404-1405.

3. Kucuk O, Ugras M, Bicer S, Col D, Giray T, Erdag GC, et al. Hyper- transaminasaemia in children with viral gastroenteritis. Infez Med 2016;24:32-37.

4. Nakajima H, Watanabe T, Miyazaki T, Takeuchi M, Honda Y, Shimada $\mathrm{N}$, et al. Acute liver dysfunction in the course of norovirus gastroenteritis. Case Rep Gastroenterol 2012;6:69-73.

5. Tsuge M, Goto S, Kato F, Morishima T. Elevation of serum transaminases with norovirus infection. Clin Pediatr (Phila) 2010;49:574-578.

6. Zenda T, Miyamoto M, Kaneko S. Norovirus gastroenteritis accompanied by marked elevation of transaminases. Hiroshima J Med Sc 2011;60:41-43.

7. Karst SM. Pathogenesis of noroviruses, emerging RNA viruses. Viruses 2010;2:748-781.

8. Echenique IA, Stosor V, Gallon L, Kaufman D, Qi C, Zembower TR. Prolonged norovirus infection after pancreas transplantation: a case report and review of chronic norovirus. Transpl Infect Dis 2016;18:98-104

9. Lee LY, Ladner DP, Ison MG. Norovirus infection in solid organ transplant recipients: a single-center retrospective study. Transpl Infect Dis 2016;18:932-938.

10. Ishak KG. Pathologic features of chronic hepatitis. A review and update. Am J Clin Pathol 2000;113:40-55.

11. Arias-Diaz J, Ildefonso JA, Muñoz JJ, Zapata A, Jiménez E. Both tacrolimus and sirolimus decrease Th1/Th2 ratio, and increase regulatory $T$ lymphocytes in the liver after ischemia/reperfusion. Lab Invest 2009:89:433-445.

12. Han G, Shao H, Peng Y, Zhang P, Ke Y, Kaplan HJ, et al. Suppressor role of rat $C D 8+C D 45 R$ Clow $T$ cells in experimental autoimmune uveitis (EAU). J Neuroimmunol 2007;183:81-88.

13. Chong PP, van Duin D, Sonderup JL, Grant WJ, Kalil AC, Florescu DF. Predictors of persistent diarrhea in norovirus enteritis after solid organ transplantation. Clin Transpl 2016;30:1488-1493.

14. Florescu DF, Hill LA, McCartan MA, Grant W. Two cases of Norwalk virus enteritis following small bowel transplantation treated with oral human serum immunoglobulin. Pediatr Transplant 2008;12:372375. 